\title{
SOME REMARKS ON SYMPLECTIC AUTOMORPHISMS
}

\section{GEORGE W. MACKEY}

1. Introduction. Let $G$ be a separable ${ }^{1}$ locally compact abelian group and let $\hat{G}$ be its dual. Let $A=G \times \hat{G}$ and let $\sigma$ be the complexvalued function on $A \times A$ defined by the equation ${ }^{2} \sigma\left(x_{1}, y_{1} ; x_{2}, y_{2}\right)$ $=y_{1}\left(x_{2}\right)\left[y_{2}\left(x_{1}\right)\right]$ - By analogy with the case in which $G$ is a finitedimensional vector space, it is natural to call an automorphism $\alpha$ of $A$ symplectic (cf. [6]) whenever $\sigma\left(\alpha\left(x_{1}, y_{1}\right) ; \alpha\left(x_{2}, y_{2}\right)\right) \equiv \sigma\left(x_{1}, y_{1} ; x_{2}, y_{2}\right)$. Let $\Sigma_{G}$ denote the group of all bicontinuous symplectic automorphisms of $A$. In the special case in which $G$ is the additive group of a locally compact field (of characteristic $\neq 2$ ) the group $\Sigma_{G}$ plays a rôle in certain unpublished ${ }^{3}$ number-theoretical investigations of $\mathrm{A}$. Weil. In a recent ${ }^{3}$ lecture at Harvard, Weil showed, among other things, that $\Sigma_{G}$ in this case admits a "natural" infinite-dimensional projective unitary representation.

In the present note we shall show first $(\$ 2)$ that this result holds for any $G$ for which $x \rightarrow x^{2}$ is an automorphism, and that the existence of the representation in question is a more or less immediate consequence of an earlier result of the author (Theorem 1 of [3]). Then in $\$ 3$ we shall show that the results of our later paper [4] yield further information. In particular, we shall show that it is possible to weaken the assumption that $x \rightarrow x^{2}$ be an automorphism and to prove that the projective representation in question is continuous in a certain sense.

I. E. Segal, who was also present at Weil's lecture, has observed that the special case in which $G$ is a real vector group occurs in his own work [5] and that of David Shale [7]. Shale's paper contains a deeper treatment of the representation than we shall attempt here. Segal is familiar with [3] and has independently observed that one can give the argument of $\$ 2$ below. He has written a note [6] in which a more complicated method is used to obtain a stronger result. Both the method and result are different from those in $\$ 3$ below.

2. The case in which $x \rightarrow x^{2}$ is an automorphism of $G$. Let $U$ be any strongly continuous unitary $\sigma$-representation of $A=G \times \hat{G}$; that is, any strongly continuous map $x, y \rightarrow U_{x, y}$, where the $U_{x, y}$ are unitary

Received by the editors March 11, 1964.

1 See $\$ 4$.

2 [ ]- denotes the complex conjugate.

- This note was written in January and February of 1963. A part of Weil's investigations have now appeared under the title Sur certaines groupes d'operateurs unitaires, Acta Math. 11 (1964), 145-211. 
operators in a separable Hilbert space and $U_{\left(x_{1}, y_{1}\right)\left(x_{2}, y_{2}\right)}=\sigma\left(x_{1}, y_{1}\right.$; $\left.x_{2}, y_{2}\right) U_{x_{1}, y_{1}} U_{x_{2}, y_{2}}$. Let $V_{x}=U_{x, e}, W_{y}=U_{e, y}$. Then $U_{x, y}=V_{x} W_{y}[y(x)]^{-}$ and $V$ and $W$ are ordinary unitary representations of $G$ and $\hat{G}$, respectively. Conversely, as a simple calculation shows, $x, y$ $\rightarrow V_{x} W_{y}[y(x)]-$ defines a strongly continuous unitary $\sigma$-representation of $A$ if and only if the following "commutation relations" hold:

$$
V_{x} W_{y}=y\left(x^{2}\right) W_{y} V_{x}
$$

If $x \rightarrow x^{2}$ is an automorphism, there exists, for each $V$, a unique strongly continuous unitary representation $\tilde{V}$ such that $\widetilde{V}_{x^{2}}=V_{x}$ for all $x$ in $G$. Clearly, $V$ and $W$ satisfy $(*)$ if and only if $\tilde{V}$ and $W$ satisfy

$$
\widetilde{V}_{x} W_{y}=y(x) W_{y} \widetilde{V}_{x} .
$$

Now, according to Theorem 1 of $[3],\left(*^{\prime}\right)$ has, to within equivalence, just one irreducible solution. Here $A$ has just one equivalence class of irreducible strongly continuous unitary $\sigma$-representations. Let $U$ be any member of this class and for each $\alpha \in \Sigma_{G}$ let $U_{x, y}^{\alpha}=U_{\alpha(x, y)}$. Then $U^{\alpha}$ is also a member of the class and, as such, is equivalent to $U$. Let $M_{\alpha}$ set up the equivalence. Then $U_{\alpha(x, y)} \equiv M_{\alpha} U_{x, y} M_{\alpha}^{-1}$ and $M_{\alpha}$ is determined up to a multiplicative constant. Since $M_{\alpha} M_{\beta}$ and $M_{\alpha \beta}$ both set up an equivalence between $U^{\alpha \beta}$ and $U$, it follows that $\alpha \rightarrow M_{\alpha}$ is a projective unitary representation of $\Sigma_{G}$.

3. Application of the results of [4]. Projective representations are studied systematically in [4] and one can obtain the uniqueness theorem which we have just applied, in addition to some further information, by specializing theorems of that paper. $\$ 8$ of [4] contains a study of how the $\sigma$-representations of a more or less general separable locally compact group $g$ are related to those of a closed normal subgroup $N$. (We now adapt the terminology of [4] and omit the words continuous and unitary in speaking of $\sigma$-representations with these properties.) Let us take $\mathcal{G}$ to be $A$ and $N$ to be $G \times e$. According to Theorem 8.4 , there is a family of irreducible $\sigma$-representations of $G$ for each orbit in a certain action of $\mathcal{G} / N$ on $\hat{N}$. In the case at hand we may identify $\mathcal{S} / N$ with $\hat{G}$ and $\hat{N}$ with $\hat{G}$. We then find that the action of $y$ in $\hat{G}$ on $y_{1}$ in $\hat{G}$ is to take it into $y_{1} y^{2}$. Thus the orbits are the cosets of the subgroup $S$ of all squares in $\hat{G}$. The equivalence classes of irreducible $\sigma$-representations associated with a fixed orbit correspond one-to-one to the equivalence classes of $\tau$-representations of the subgroup of $g / N$ leaving a point of the orbit fixed. Here $\tau$ is a multiplier described in the proof of Theorem 8.2.

Suppose that $x \rightarrow x^{2}$ is an automorphism of $G$ so that $y \rightarrow y^{2}$ is an 
automorphism of $\hat{G}$. Then $S=\hat{G}$ and there is only one orbit. Moreover, the relevant subgroup of $\mathcal{G} / N=\hat{G}$ is the identity so there can be only one equivalence class of $\tau$-representations. Since $S$ is closed, Theorem 9.1 applies and tells us that there are no irreducible $\sigma$ representations except those described by Theorem 8.4. Thus $A$ has only one equivalence class of $\sigma$-representations whenever $x \rightarrow x^{2}$ is an automorphism of $G$.

To see what $\$ \$ 8$ and 9 of [4] tell us in more general cases, let us first observe that the multiplier $\tau$ is identically one whenever $\mathcal{G}=A, N$ $=G \times e$ and $\sigma$ is as above. It follows that the equivalence classes of irreducible $\sigma$-representations associated with each $S$ coset correspond one-to-one to the members of the character group $\hat{S}^{\prime}$ of the group $S^{\prime}$ of all elements of order 2 in $\hat{G}$. When $S$ is closed, it follows from Theorem 9.1 that the irreducible $\sigma$-representations described in Theorem 8.4 are exhaustive. Though we shall not do so here, the considerations of $\$ 5$ of [4] can be extended to prove that $A$ has further irreducible $\sigma$-representations whenever $S$ is not closed. Thus we have

TheOREM 1. The group $A=G \times \hat{G}$ has just one equivalence class of irreducible $\sigma$-representations if and only if $x \rightarrow x^{2}$ is an automorphism of $G$. If $x \rightarrow x^{2}$ is not an automorphism of $G$ then there is a "natural" one-to-one map of $(\hat{G} / S) \times \hat{S}^{\prime}$ into the set of equivalence classes of irreducible $\sigma$-representations of $A$. Here, $S$ is the group of all squares in $\hat{G}$ and $S^{\prime}$ is the group of all elements of order 2 in $\hat{G}$. This map is "onto" if and only if $S$ is closed in $\hat{G}$.

We now reformulate the one-to-one correspondence described in Theorem 1 in such a way that the natural action of $\Sigma_{G}$ on the irreducible $\sigma$-representations of $A$ can be studied. The notion of "induced representation" is defined for $\sigma$-representations on p. 274 of [4] and, by Theorem 8.4 of that paper, the $\sigma$-representations of $A$ described in Theorem 1 above are all induced by one-dimensional $\sigma$-representations of the subgroup $G \times S^{\prime}$ of $A$. The mapping $x, \eta \rightarrow \sigma(x, e ; e, \eta)$ $=[\eta(x)]^{-}=\eta(x)$ is one such and the most general one is $x, \eta$ $\rightarrow \eta(x) y_{1}(x) z_{1}(\eta)$, where $y_{1} \in \hat{G}_{1}$ and $z_{1} \in \hat{S}^{\prime}$. Let $U^{y_{1}, z_{1}}$ denote the $\sigma$ representation of $A$ induced by $x, \eta \rightarrow \eta(x) y_{1}(x) z(\eta)$. By Theorem 8.4, $U^{y_{1}, z_{1}}$ is irreducible and $U^{y_{1}, z_{1}}$ is equivalent to $U^{y_{2}, z_{2}}$ if and only if $y_{1}$ and $y_{2}$ lie in the same $S$ coset. The resulting one-to-one map of $\hat{G} / S \times \hat{S}^{\prime}$ into irreducible $\sigma$-representations is the one alluded to in Theorem 1. Let $S^{\perp}$ denote the annihilator of $S$ in $G$, that is, the group of all elements of order 2 in $G$. Then $S^{\perp} \times S^{\prime}$ is just the subgroup of all elements of order 2 in $A$ and as such can be described without reference to the factorization $A=G \times \hat{G}$. The restriction to $S^{\perp} \times S^{\prime}$ of the 
$\sigma$-representation $U^{v_{1}, z_{1}}$ is easily computed to be a multiple of the onedimensional $\sigma$-representation $\xi, \eta \rightarrow \eta(\xi) y_{1}(\xi) z_{1}(\eta)$. Moreover, it follows from Theorem 7.1 of [4] that any irreducible $\sigma$-representation of $A$ which is not equivalent to one of the $U^{v_{1}, z_{1}}$ must have a restriction to $S^{\perp} \times S^{\prime}$ which is a direct integral of infinitely many distinct one-dimensional $\sigma$-representations. We can now conclude the truth of the following variant of Theorem 1 .

THEOREM $1^{\prime}$. Let $A_{0}$ be the subgroup of $A=G \times \hat{G}$ consisting of all elements of $A$ of order 2 . Then, for each member $X$ of $\hat{A}_{0}$, there exists (to within equivalence) just one irreducible $\sigma$-representation $W^{\mathbf{x}}$ of $A$ whose restriction to $A_{0}$ is a multiple of the one-dimensional $\sigma$-representation $\xi, \eta \rightarrow \eta(\xi) \mathrm{X}(\xi, \eta)$. The $\sigma$-representations $W^{\mathrm{X}}$ will exhaust the irreducible $\sigma$-representations of $A$ if and only if $S$ is a closed subgroup of $\hat{G}$.

It is clear that each $\alpha \in \Sigma_{G}$ carries $A_{0}$ onto $A_{0}$ and every irreducible $\sigma$-representation of the form $W^{\mathrm{X}}$ into another of the same form $W_{\alpha(x, y)}^{\mathrm{X}} \equiv W_{x, y}^{\mathrm{X} \alpha}$. However, it is not clear that the mapping $\mathrm{X} \rightarrow \mathrm{X}^{\alpha}$ is that induced by the action of $\alpha$ on $A_{0}$. Let $\phi_{0}(\xi, \eta)=\eta(\xi)$. Then $\mathrm{X}^{\alpha}(\xi, \eta)=\mathrm{X}(\alpha(\xi, \eta)) \phi_{0}(\alpha(\xi, \eta)) / \phi_{0}(\xi, \eta)$. Thus, if we restrict $\alpha$ to lie in the subgroup $\Sigma_{G}^{0}$ of $\Sigma_{G}$ consisting of all $\alpha$ with $\phi_{0}(\alpha(\xi, \eta)) \equiv \phi_{0}(\xi, \eta)$, then $\mathrm{X}^{\alpha}(\xi, \eta) \equiv \mathrm{X}(\alpha(\xi, \eta))$ and, taking $x$ to be the identity character, we conclude the truth of

ThEOREM 2. Let $W$ be the unique irreducible $\sigma$-representation of $A=G \times \hat{G}$ which is a multiple of the one-dimensional $\sigma$-representation $\xi, \eta \rightarrow \eta(\xi)$ when restricted to $A_{0}=S^{\perp} \times S^{\prime}$. Then, for every $\alpha$ in $\Sigma_{G}^{0}$, the $\sigma$-representation $x, y \rightarrow W_{\alpha(x, y)}$ of $A$ is equivalent to $W$.

If we replace the unique $\sigma$-representation of $\$ 2$ by $W$ and replace $\Sigma_{G}$ by $\Sigma_{G}^{0}$ we may define a projective representation $M$ just as we did at the end of $\$ 2$. However, we now need make no restriction on the endomorphism $x \rightarrow x^{2}$. Note that $\Sigma_{G}^{0}=\Sigma_{G}$ whenever either $S^{\perp}$ or $S^{\prime}$ reduces to the identity.

We complete this section with the promised result about continuity.

THEOREM 3. Let $\Sigma$ be a subgroup of $\Sigma_{G}^{0}$ topologized so as to be separable, locally compact and such that $x, y, \alpha \rightarrow \alpha(x, y)$ is continuous from $A \times \Sigma$ to $A$. Then the projective representation $M$ of $\Sigma_{G}^{0}$, defined as indicated above, is continuous when restricted to $\Sigma$ in the sense that $\alpha \rightarrow\left|M_{\alpha}(\phi) \cdot \psi\right|$ is a continuous function of $\alpha$ for all $\phi$ and $\psi$ in the Hilbert space.

Proof. Let $g$ be the group of all triples $x, y, \alpha$ with $x \in G, y \in \hat{G}$, 
$\alpha \in \Sigma$. Make $g$ into a separable locally compact group by setting $\left(x_{1}, y_{1}, \alpha_{1}\right)\left(x_{2}, y_{2}, \alpha_{2}\right)=\left(\left(x_{1}, y_{1}\right) \alpha\left(x_{2}, y_{2}\right), \alpha_{1} \alpha_{2}\right)$. Let $W$ be the irreducible $\sigma$-representation of $G \times \hat{G}$ described in Theorem 2 . Let $\sigma^{\prime}$ be the multiplier for $G$ defined as follows.

$$
\sigma^{\prime}\left(x_{1}, y_{1}, \alpha_{1} ; x_{2}, y_{2}, \alpha_{2}\right)=\sigma\left(x_{1}, y_{1}, \alpha_{1}\left(x_{2}, y_{2}\right)\right) \text {. }
$$

Apply Theorem 8.2 of [4] with $\pi=G \times \hat{G} \times e, L=W$. Then our $M$ is just the restriction to $e \times \Sigma$ of the $M$ of that theorem. As such it is continuous in the sense indicated.

4. On avoiding the hypothesis of separability. Loomis in [1] has generalized the main theorem of [3] to the inseparable case. Using this generalization we see at once that the discussion of $\$ 2$ does not really require the separability hypothesis. The extent to which separability may be avoided in $\$ 3$ is not clear. Loomis's paper [2] shows that one of the key results of [4] is valid for nonseparable groups but a close examination of the arguments of [4] would have to be made before one could say whether or not separability is needed elsewhere.

\section{BIBLIOGRAPHY}

1. L. H. Loomis, Note on a theorem of Mackey, Duke Math. J. 19 (1952), 641-645.

2. - Positive definite functions and induced representations, Duke Math. J. 27 (1960), 564-579.

3. G. W. Mackey, On a theorem of Stone and von Neumann, Duke Math. J. 16 (1949), 313-326.

4. - Unitary representations of group extensions. I, Acta Math. 99 (1958), 265-311.

5. I. E. Segal, Foundations of the theory of dynamical systems of infinitely many degrees of freedom. I, Mat. Fys. Medd. Danske Vid. Selsk. 31 (1959), no. 12, 1-39.

6. - Transforms for operators and symplectic automorphisms over a locally compact abelian group, Math. Scand. 13 (1963), 31-43.

7. D. Shale, Linear symmetries of free boson fields, Trans. Amer. Math. Soc. 103 (1962), 149-167.

\section{HARVARD UNIVERSITY}

